\title{
SISTEMATIZAÇÃO DA ASSISTÊNCIA DE ENFERMAGEM NO PERIODO PERIOPERATÓRIO: ANÁLISE DE REGISTROS
}

\author{
Nursing care systematization in the perioperative period: analysis of records
}

Sistematización de la asistencia de enfermería en el periodo perioperativo: análisis de registros

Franciele Cristine Fengler ${ }^{1}$ (D) Cássia Regina Gotler Medeiros*

RESUMO: Objetivo: Analisar os registros da sistematização da assistência de enfermagem perioperatória (SAEP) conforme recomendações da Associação Brasileira de Enfermeiros de Centro Cirúrgico, Recuperação Anestésica e Centro de Material e Esterilização (SOBECC). Método: Estudo descritivo, documental e quantitativo desenvolvido em agosto de 2018 em um hospital do Rio Grande do Sul. A amostra foi composta por 50 prontuários de pacientes submetidos a procedimentos anestésico-cirúrgicos, selecionados aleatoriamente em uma unidade de internação cirúrgica. O instrumento de pesquisa coletou dados referentes a 10 atributos, subdivididos entre as fases do perioperatório, conforme as práticas recomendadas pela SOBECC. Os resultados estão apresentados por frequências absolutas e relativas. O estudo foi aprovado pelo Comitê de Ética em Pesquisa da instituição. Resultados: O maior percentual de registros foi totalmente atingido (61,40\%). Porém verificou-se que $25,79 \%$ dos registros não foram realizados, principalmente aqueles relacionados aos cuidados da enfermagem. Conclusão: Conforme as recomendações da SOBECC, observou-se deficiência nos registros e na adesão à SAEP. Palavras-chave: Assistência de enfermagem. Enfermagem perioperatória. Qualidade da assistência à saúde.

ABSTRACT:Objective: To analyze records on the perioperative nursing care systematization (PNCS) according to recommendations of the Brazilian Society of Surgical, Anesthesia Recovery, Sterilization and Material Center Nurses (SOBECC). Method: Descriptive, documentary, and quantitative study developed in August 2018 on a hospital in the state of Rio Grande do Sul. The sample consisted of 50 medical records of patients who underwent anesthetic-surgical procedures, randomly selected from a surgical inpatient unit. Using the research instrument, we collected data on ten attributes, subdivided into perioperative phases, according to the practices recommended by SOBECC. The results are expressed as absolute and relative frequencies. The study was approved by the Research Ethics Committee of the institution. Results: The highest percentage of records was fully complied with (61.40\%). However, $25.79 \%$ of records were not followed, especially those related to nursing care. Conclusion: According to SOBECC's recommendations, records and adherence to PNCS were inadequate.

Keywords: Nursing care. Perioperative nursing. Quality of health care.

RESUMEN: Objetivo: Analizar los registros de la sistematización de la atención de enfermería perioperatoria (SAEP) según lo recomendado por la Asociación Brasileña de Enfermeras del Centro Quirúrgico, Centro de Recuperación Anestésica y Material y Esterilización (SOBECC). Método: Estudio descriptivo, documental y cuantitativo desarrollado en agosto de 2018 en un hospital de Rio Grande do Sul. La muestra consistió en 50 registros médicos de pacientes sometidos a procedimientos anestésico-quirúrgicos, seleccionados al azar en una unidad de pacientes internados quirúrgicos. El instrumento de investigación recolectó datos referentes a 10 atributos, subdivididos entre las fases perioperatorias, de acuerdo con las prácticas recomendadas por SOBECC. Los resultados se presentan por frecuencias absolutas y relativas. El estudio fue aprobado por el Comité de Ética en Investigación de la institución. Resultados: El porcentaje más alto de registros se logró completamente (61,40\%). Sin embargo, se encontró que el 25,79\% de los registros no se hicieron, especialmente aquellos relacionados con la atención de enfermería. Conclusión: De acuerdo con las recomendaciones de SOBECC, hubo una deficiencia en los registros y el cumplimiento de SAEP.

Palabras clave: Atención de enfermería. Enfermería perioperatoria. Calidad de la atención de salud.

'Graduada em Enfermagem. Prestadora de serviços de enfermagem autônoma - Venâncio Aires (RS), Brasil.

2Doutora em Enfermagem pela Universidade Federal do Rio Grande do Sul (UFRGS). Professora titular na Univates - Lajeado (RS), Brasil.

*Autor correspondente: cgotlermedeiros@gmail.com

Recebido: 12/02/2019 - Aprovado: 20/11/2019

DOI: $10.5327 / Z 1414-4425202000010008$ 


\section{INTRODUÇÃo}

$\mathrm{Na}$ assistência ao paciente, a enfermagem segue um instrumento metodológico denominado de processo de enfermagem (PE). Esse instrumento sistemático e humanizado é utilizado para nortear o cuidado de enfermagem ${ }^{1}$. A operacionalização do $\mathrm{PE}$ ocorre no momento em que se implementa a sistematização da assistência de enfermagem (SAE), tornando o processo de trabalho mais eficiente. No período perioperatório, que envolve toda a experiência cirúrgica do paciente, o PE é chamado de sistematização da assistência de enfermagem perioperatória $(\mathrm{SAEP})^{2}$.

$\mathrm{O} P \mathrm{PE}$ é considerado um instrumento metodológico que orienta o cuidado de enfermagem e a documentação do exercício profissional, contribuindo para melhorar a qualidade da assistência e aumentar a visibilidade e o reconhecimento profissional. Esse instrumento compõe-se de cinco etapas: histórico de enfermagem/ coleta de dados, diagnóstico de enfermagem, prescrição/planejamento da assistência de enfermagem, implementação da assistência e evolução/ avaliação de enfermagem ${ }^{1}$.

A SAEP é um modelo que promove a interação da assistência entre os períodos pré, trans e pós-operatório, possibilitando o planejamento e o controle em cada fase do desenvolvimento da assistência operatória. Sustenta as ações de enfermagem no centro cirúrgico (CC) com o propósito de assistir ao paciente e à família de forma integral, tendo em vista uma assistência de enfermagem de qualidade ${ }^{1}$. Além disso, promove uma intervenção adequada, planejada e fundamentada, voltada aos problemas de cada paciente no perioperatório, bem como à avaliação dos resultados ${ }^{3}$.

O período perioperatório, conforme a Associação Brasileira de Enfermeiros de Centro Cirúrgico, Recuperação Anestésica e Centro de Material e Esterilização (SOBECC) ${ }^{1}$, pode ser definido como o intervalo de tempo que compreende as atividades desenvolvidas em cada período cirúrgico. Divide-se em:

- Pré-operatório mediato: inicia-se no momento da definição da cirurgia e estende-se até 24 horas antes da realização do procedimento;

- pré-operatório imediato: começa 24 horas antes do procedimento cirúrgico e abrange até o momento em que o paciente é recebido no CC;

- transoperatório: do momento em que o paciente é recebido no CC até sua saída da sala de operação;

- intraoperatório: está inserido no transoperatório, iniciando-se com o procedimento anestésico-cirúrgico e estendendo-se até o seu término;
- pós-operatório: fase que compreende todo o período após o ato anestésico-cirúrgico, subdividindo-se em três momentos - recuperação anestésica, da chegada do paciente à sala de recuperação pós-anestésica (SRPA) até sua alta para unidade de origem; pós-operatório imediato, do término do procedimento anestésico-cirúrgico até 24 horas depois; e pós-operatório mediato, após as primeiras 24 horas do procedimento anestésico-cirúrgico até a alta hospitalar ou o retorno do paciente ao seu domicílio.

A avaliação da assistência de enfermagem perioperatória deve ser efetuada ao término de cada período do desenvolvimento cirúrgico, considerando a satisfação do paciente, as atividades realizadas pelo enfermeiro e o desempenho do trabalho da equipe, conforme o modelo de assistência definido pela equipe e pela instituição ${ }^{4}$.

Os profissionais, em sua maioria, acreditam que a SAEP seja indispensável para um atendimento de qualidade aos pacientes, entretanto enfrentam dificuldades para implantá-la. Tais dificuldades estão relacionadas à falta de tempo, à sobrecarga de trabalho e à equipe administrativa, que, por vezes, não compreende a importância da atuação do enfermeiro na assistência ao paciente no perioperatório, desviando o profissional da sua função assistencial para a gerencial ${ }^{2,5,6}$.

Estudos indicam que a qualidade da assistência de enfermagem desempenhada no período perioperatório interfere no resultado do ato anestésico-cirúrgico. Dessa forma, busca-se entender o exercício da enfermagem em uma unidade de internação cirúrgica, sinalizando sua relevância para a qualidade da assistência à saúde ${ }^{2}$.

No Brasil, mesmo com as recomendações da SOBECC e da Association of periOperative Registered Nurses (AORN) no que tange à adoção de um modelo de assistência, a fim de nortear ações dos enfermeiros no CC, a maior parte dos hospitais ainda não adotou um modelo formal. Utiliza-se o planejamento baseado na programação cirúrgica, em que o enfermeiro gerencia os recursos materiais e humanos para a previsão e a provisão do ato anestésico-cirúrgico, no entanto a maior crítica a esse modelo é a falta de registros, prejudicando o planejamento da assistência individualizada e a adequação dos recursos humanos e materiais para a realização do procedimento anestésico-cirúrgico. Além disso, a falta de registros deslegitima o trabalho desenvolvido pela equipe de enfermagem e não respalda o enfermeiro em casos de ocorrências jurídicas ${ }^{4}$.

Estudos demonstram que, mesmo que a legislação ressalte a importância dos registros de enfermagem para a 
documentação e o amparo da profissão, os profissionais, embora cientes de tal condição, não realizam os registros com qualidade, nem os consideram instrumento de trabalho, dificultando sua funcionalidade ${ }^{7}$.

Segundo pesquisadores, são escassos os estudos relacionados à avaliação do paciente e da assistência perioperatória prestada, bem como de instrumentos de registros e da percepção dos pacientes ${ }^{6}$. Assim, este estudo levanta a questão: como está sendo utilizada a SAE no período perioperatório?

\section{OBJETIVO}

Analisar os registros da SAE realizados no período perioperatório à luz das recomendações da SOBECC.

\section{MÉTODO}

Este estudo é de caráter descritivo e documental, tem abordagem quantitativa e foi desenvolvido no ano de 2018 com base na análise de registros de enfermagem em prontuários de pacientes submetidos a procedimentos anestésico-cirúrgicos variados em um hospital do interior do Rio Grande do Sul. Trata-se de uma instituição filantrópica com 138 leitos que atende $70 \%$ de seus pacientes via Sistema Único de Saúde (SUS).

Os prontuários foram selecionados de forma aleatória em uma unidade de internação cirúrgica, após a alta hospitalar do paciente. Na instituição são realizadas ao mês, em média, 259 cirurgias, e ao dia, em média, oito cirurgias. Assim, para o estudo, foram selecionados $20 \%$ dos prontuários das cirurgias feitas no mês de agosto de 2018, compondo uma amostra de 50 prontuários. Incluíram-se prontuários de pacientes com idade acima de 20 anos, de ambos os sexos, submetidos a procedimentos anestésico-cirúrgicos variados, de médio e grande porte. Foram excluídos procedimentos ambulatoriais.

Coletaram-se os dados por meio da aplicação de um instrumento elaborado pelos próprios pesquisadores. $\mathrm{O}$ instrumento contém 10 atributos, subdivididos entre as fases do perioperatório, conforme as práticas recomendadas pelas diretrizes da SOBECC ${ }^{1}$.

Classificaram-se os atributos em quatro categorias: totalmente atingidos $(\mathrm{TA})$, parcialmente atingidos $(\mathrm{PA})$, não atingidos (NA) e não se aplica (NSA). A categoria NSA foi utilizada somente para os casos em que não foi necessária a realização do respectivo procedimento. Como o estudo é documental, foi considerado atingido somente o que estava registrado. Em planilhas do Microsoft Office Excel 2013, realizou-se a análise da frequência absoluta e relativa dos atributos, que está apresentado na forma de tabelas.

O estudo foi desenvolvido após autorização do local da pesquisa mediante a carta de anuência e a aprovação do Comitê de Ética em Pesquisa, sob o $\mathrm{n}^{\circ}$ Certificado de apresentação para Apreciação Ética (CAAE) 93498218.1.0000.5310, em conformidade com a Resolução do Conselho Nacional de Saúde $\mathrm{n}^{\circ} 466 / 2012$, que regulamenta as pesquisas envolvendo seres humanos ${ }^{8}$. Foram respeitados os aspectos éticos quanto ao sigilo da identificação da instituição e dos pacientes, por ocasião da divulgação dos resultados do estudo.

\section{RESULTADOS}

O período pré-operatório contempla quatro atributos. Apesar de em todos os atributos a maior frequência encontrada ter sido de TA, observa-se que os registros do histórico e dos diagnósticos de enfermagem foram realizados em pouco mais de $50 \%$ dos prontuários, conforme Tabela $1 . \mathrm{O}$ atributo

Tabela 1. Frequência dos registros de atributos do período pré-operatório.

\begin{tabular}{|c|c|c|c|c|c|c|c|c|}
\hline \multirow{2}{*}{ Procedimentos } & \multicolumn{2}{|c|}{ TA } & \multicolumn{2}{|c|}{ PA } & \multicolumn{2}{|c|}{ NA } & \multicolumn{2}{|c|}{ NSA } \\
\hline & $\mathbf{N}$ & $\%$ & $\mathbf{N}$ & $\%$ & $\mathbf{N}$ & $\%$ & $\mathbf{N}$ & $\%$ \\
\hline 1. Histórico & 26 & 52 & 3 & 6 & 21 & 42 & 0 & 0 \\
\hline 2. Exame físico & 44 & 88 & 6 & 12 & 0 & 0 & 0 & 0 \\
\hline 3. Identificação dos diagnósticos de enfermagem & 26 & 52 & 0 & 0 & 24 & 48 & 0 & 0 \\
\hline $\begin{array}{l}\text { 4. Realização da prescrição de enfermagem para o pré e } \\
\text { transoperatório }\end{array}$ & 31 & 62 & 0 & 0 & 19 & 38 & 0 & 0 \\
\hline Total & 127 & 63,5 & 9 & 4,5 & 64 & 32 & 0 & 0 \\
\hline
\end{tabular}

TA: totalmente atingido; PA: parcialmente atingido; NA: não atingido; NSA: não se aplica. 
registrado com maior frequência foi o exame físico (88\%). O total de atributos registrados nesse período ficou em $63,50 \%$.

O transoperatório é composto do atributo 5, com nove procedimentos, do atributo 6 , com 15 procedimentos, e do atributo 7 , com um procedimento. Os registros TA foram encontrados em $57,44 \%$ dos prontuários, de acordo com a Tabela 2. No atributo 5 , todos os procedimentos (100\%) foram registrados.

O atributo 6, que se refere à evolução de enfermagem, foi o de menor frequência de registro, atingindo 47,06\%. Nesse atributo, os procedimentos com registros NA em mais de $80 \%$ dos prontuários foram: posição do paciente durante a anestesia e a cirurgia, uso de coxins de proteção, tipo de aquecimento utilizado e locais de monitorização e de colocação da placa de eletrocautério. Verificou-se que os registros de maior frequência (100\%) se concentraram nos procedimentos de maior interesse para os médicos e para a instituição, como: tipo de anestesia, horário de início e término do procedimento anestésico-cirúrgico, registro de materiais e equipamentos utilizados durante o procedimento anestésico-cirúrgico, entre outros.
O registro do sétimo atributo, início da prescrição de enfermagem pós-operatória ao término da cirurgia, foi NA em $100 \%$ dos prontuários.

O pós-operatório abrange os atributos 8 (com um procedimento), 9 (com seis procedimentos) e 10 (com um procedimento), conforme a Tabela 3. Nesse período, $72,75 \%$ dos registros foram TA, no entanto o décimo atributo foi $100 \% \mathrm{NA}$

Embora no período transoperatório para o sétimo atributo, acerca do início da prescrição de enfermagem pós-operatória ao término da cirurgia, os registros tenham sido NA em $100 \%$ dos casos, consideraram-se, em relação ao atributo 8 , os registros referentes à prescrição de enfermagem pós-operatória, ainda que não tenham sido continuidade da mesma prescrição.

Haja vista todo o período perioperatório, verificou-se que o maior percentual nos prontuários analisados foi de TA $(61,40 \%)$, conforme a Tabela 4 , no entanto $25,79 \%$ dos registros não foram realizados, principalmente aqueles relacionados aos cuidados específicos da enfermagem.

Tabela 2. Frequência dos registros de atributos do período transoperatório.

\begin{tabular}{|c|c|c|c|c|c|c|c|c|}
\hline \multirow{2}{*}{ Procedimentos } & \multicolumn{2}{|c|}{ TA } & \multicolumn{2}{|c|}{ PA } & \multicolumn{2}{|c|}{ NA } & \multicolumn{2}{|c|}{ NSA } \\
\hline & $\mathbf{N}$ & $\%$ & $\mathbf{N}$ & $\%$ & $\mathbf{N}$ & $\%$ & $\mathbf{N}$ & $\%$ \\
\hline \multicolumn{9}{|l|}{ 5. Implementação da assistência } \\
\hline Recepção e identificação do paciente no CC & 50 & 100 & 0 & 0 & 0 & 0 & 0 & 0 \\
\hline Aferição dos sinais vitais & 50 & 100 & 0 & 0 & 0 & 0 & 0 & 0 \\
\hline Realização do exame físico simplificado & 50 & 100 & 0 & 0 & 0 & 0 & 0 & 0 \\
\hline Realização do checklist & 50 & 100 & 0 & 0 & 0 & 0 & 0 & 0 \\
\hline $\begin{array}{l}\text { Colocação da placa de eletrocautério no local } \\
\text { apropriado }\end{array}$ & 50 & 100 & 0 & 0 & 0 & 0 & 0 & 0 \\
\hline $\begin{array}{l}\text { Controle de perdas sanguíneas, diurese e secreção } \\
\text { gástrica, quando o paciente estiver com SNG aberta } \\
\text { durante a cirurgia }\end{array}$ & 50 & 100 & 0 & 0 & 0 & 0 & 0 & 0 \\
\hline Realização de cateterismo vesical quando necessário & 10 & 20 & 0 & 0 & 0 & 0 & 40 & 80 \\
\hline $\begin{array}{l}\text { Identificação e encaminhamento de peça } \\
\text { anatomopatológica }\end{array}$ & 5 & 10 & 0 & 0 & 0 & 0 & 45 & 90 \\
\hline $\begin{array}{l}\text { Registro de todos os cuidados de enfermagem } \\
\text { prestados ao paciente }\end{array}$ & 50 & 100 & 0 & 0 & 0 & 0 & 0 & 0 \\
\hline Total & 365 & 81,11 & 0 & 0 & 0 & 0 & 85 & 18,89 \\
\hline \multicolumn{9}{|l|}{ 6. Evolução de enfermagem deve apresentar } \\
\hline $\begin{array}{l}\text { Identificação do paciente, da equipe cirúrgica, de } \\
\text { circulante de sala e do enfermeiro }\end{array}$ & 3 & 6 & 33 & 66 & 14 & 28 & 0 & 0 \\
\hline $\begin{array}{l}\text { Horário de início e término do procedimento } \\
\text { anestésico-cirúrgico }\end{array}$ & 50 & 100 & 0 & 0 & 0 & 0 & 0 & 0 \\
\hline
\end{tabular}


Tabela 2. Continuação.

\begin{tabular}{|c|c|c|c|c|c|c|c|c|}
\hline \multirow{2}{*}{ Procedimentos } & \multicolumn{2}{|c|}{ TA } & \multicolumn{2}{|c|}{ PA } & \multicolumn{2}{|c|}{ NA } & \multicolumn{2}{|c|}{ NSA } \\
\hline & $\mathbf{N}$ & $\%$ & $\mathbf{N}$ & $\%$ & $\mathbf{N}$ & $\%$ & $\mathbf{N}$ & $\%$ \\
\hline Nome da cirurgia & 37 & 74 & 0 & 0 & 13 & 26 & 0 & 0 \\
\hline Tipo de anestesia & 50 & 100 & 0 & 0 & 0 & 0 & 0 & 0 \\
\hline Posição do paciente durante a anestesia e cirurgia & 10 & 20 & 0 & 0 & 40 & 80 & 0 & 0 \\
\hline Uso de coxins de proteção & 1 & 2 & 0 & 0 & 49 & 98 & 0 & 0 \\
\hline Tipo de aquecimento utilizado & 0 & 0 & 0 & 0 & 50 & 100 & 0 & 0 \\
\hline $\begin{array}{l}\text { Locais de monitorização e de colocação da placa de } \\
\text { eletrocautério }\end{array}$ & 4 & 8 & 0 & 0 & 46 & 92 & 0 & 0 \\
\hline $\begin{array}{l}\text { Locais das punções venosas, arteriais, drenos, } \\
\text { cateteres, tipo de sondagens }\end{array}$ & 50 & 100 & 0 & 0 & 0 & 0 & 0 & 0 \\
\hline Encaminhamento de peça e exames ao laboratório & 7 & 14 & 0 & 0 & 0 & 0 & 43 & 86 \\
\hline Intercorrências, se houver & 8 & 16 & 0 & 0 & 0 & 0 & 42 & 84 \\
\hline Perdas sanguíneas e transfusões & 8 & 16 & 0 & 0 & 0 & 0 & 42 & 84 \\
\hline $\begin{array}{l}\text { Na ficha própria da instituição os indicadores } \\
\text { multiparamétricos garantindo que o material } \\
\text { utilizado estava esterilizado }\end{array}$ & 50 & 100 & 0 & 0 & 0 & 0 & 0 & 0 \\
\hline $\begin{array}{l}\text { Em caso de implantes, na ficha, os códigos referentes } \\
\text { ao produto colocado e prazo de validade }\end{array}$ & 25 & 50 & 0 & 0 & 0 & 0 & 25 & 50 \\
\hline $\begin{array}{l}\text { Registro de materiais e equipamentos utilizados } \\
\text { durante o procedimento anestésico-cirúrgico }\end{array}$ & 50 & 100 & 0 & 0 & 0 & 0 & 0 & 0 \\
\hline Total & 353 & 47,06 & 33 & 4,40 & 212 & 28,27 & 152 & 20,27 \\
\hline $\begin{array}{l}\text { 7. Início da prescrição de enfermagem pós-operatória } \\
\text { ao término da cirurgia }\end{array}$ & 0 & 0 & 0 & 0 & 50 & 100 & 0 & 0 \\
\hline Total geral & 718 & 57,44 & 33 & 2,64 & 262 & 20,96 & 237 & 18,96 \\
\hline
\end{tabular}

TA: totalmente atingido; PA: parcialmente atingido; NA: não atingido; NSA: não se aplica; CC: centro cirúrgico; SNG: sonda nasogástrica.

Tabela 3. Frequência dos registros de atributos do período pós-operatório.

\begin{tabular}{|c|c|c|c|c|c|c|c|c|}
\hline \multirow{2}{*}{ Procedimentos } & \multicolumn{2}{|c|}{ TA } & \multicolumn{2}{|c|}{ PA } & \multicolumn{2}{|c|}{ NA } & \multicolumn{2}{|c|}{ NSA } \\
\hline & $\mathbf{N}$ & $\%$ & $\mathbf{N}$ & $\%$ & $\mathbf{N}$ & $\%$ & $\mathbf{N}$ & $\%$ \\
\hline $\begin{array}{l}\text { 8. Continuidade da prescrição de enfermagem } \\
\text { pós-operatória }\end{array}$ & 24 & 48 & 0 & 0 & 26 & 52 & 0 & 0 \\
\hline 9. Verificação das condições clínicas do paciente & & & & 0 & & & 0 & 0 \\
\hline Entrevista & 29 & 58 & 0 & 0 & 21 & 42 & 0 & 0 \\
\hline Exame físico & 38 & 76 & 0 & 0 & 12 & 24 & 0 & 0 \\
\hline Aferição dos sinais vitais & 50 & 100 & 0 & 0 & 0 & 0 & 0 & 0 \\
\hline $\begin{array}{l}\text { Verificação das condições de curativos, acesso } \\
\text { venoso e sondas ou cateteres }\end{array}$ & 50 & 100 & 0 & 0 & 0 & 0 & 0 & 0 \\
\hline Controle da ingesta hídrica e alimentar & 50 & 100 & 0 & 0 & 0 & 0 & 0 & 0 \\
\hline Observação das eliminações vesicais e intestinais & 50 & 100 & 0 & 0 & 0 & 0 & 0 & 0 \\
\hline $\begin{array}{l}\text { 10. Realização da avaliação da assistência prestada } \\
\text { verificando a necessidade ou o aprimoramento em } \\
\text { alguma conduta e da realização da SAEP }\end{array}$ & 0 & 0 & 0 & 0 & 50 & 100 & 00 & 00 \\
\hline Total & 291 & 72,75 & 0 & 0 & 109 & 27,25 & 0 & 0 \\
\hline
\end{tabular}


Tabela 4. Frequência dos registros dos atributos do período perioperatório.

\begin{tabular}{|l|c|c|c|c|c|}
\hline Período Perioperatório & $\begin{array}{c}\text { TA } \\
(\%)\end{array}$ & $\begin{array}{c}\text { PA } \\
(\%)\end{array}$ & $\begin{array}{c}\text { NA } \\
(\%)\end{array}$ & $\begin{array}{c}\text { NSA } \\
(\%)\end{array}$ & N \\
\hline Total & 61,40 & 2,27 & 23,52 & 12,81 & 50 \\
\hline
\end{tabular}

TA: totalmente atingido; PA: parcialmente atingido; NA: não atingido; NSA: não se aplica.

\section{DISCUSSÃO}

Os resultados evidenciaram que no período pré-operatório, ainda que o percentual de atributos TA tenha ocorrido com maior frequência, houve grande deficiência e necessidade de melhora nos registros da assistência de enfermagem, uma vez que esse período se refere ao início do perioperatório, que envolve a acolhida do paciente e da família, a formação de vínculo e o preparo para o procedimento anestésico-cirúrgico.

A visita pré-operatória de enfermagem representa o princípio da realização da SAEP, contribui para a satisfação das necessidades físicas e emocionais do paciente e diminui níveis de estresse e ansiedade, auxiliando na superação do trauma cirúrgico, na recuperação e no retorno do bem-estar. Além disso, proporciona a formação do vínculo entre profissional, paciente e família, permitindo uma assistência sistematizada e contínua, voltada para cada indivíduo de forma integral e individualizada, respeitando valores, experiências e expectativas ${ }^{9}$. No transoperatório, as ações assistenciais devem ser desenvolvidas por toda a equipe de enfermagem, atendendo às atividades e expectativas do paciente, transmitindo apoio e atenção, respeitando suas crenças e valores, medos e necessidades com segurança, destreza e eficácia ${ }^{1}$.

Os resultados sinalizam a deficiência dos registros de enfermagem de forma geral, principalmente nos seguintes atributos: o posicionamento e a proteção do paciente na mesa cirúrgica, o aquecimento e a manutenção da temperatura, a monitorização e locais de colocação da placa do eletrocautério e a identificação do paciente e da equipe. Além disso, ficou evidente a ausência da prescrição de enfermagem pós-operatória ao término do ato anestésico-cirúrgico. Esses procedimentos são fundamentais para que se obtenha um período cirúrgico bem-sucedido e com segurança, sendo responsabilidade de todos os membros da equipe. Para tal, os profissionais devem estar atentos, identificar riscos e manter a segurança do paciente, protegendo-o de traumas e possíveis eventos adversos ${ }^{1}$.

Cabe a toda equipe envolvida no cuidado ao paciente cirúrgico a adoção de medidas de prevenção aos eventos citados. Os enfermeiros devem estar cientes quanto às complicações, identificando aqueles pacientes que apresentam maiores riscos na avaliação pré-operatória de enfermagem, uma vez que as medidas de precaução podem diminuir os riscos de complicações associadas, que envolvem infecção de sítio cirúrgico, sangramento no decorrer da cirurgia por conta de distúrbios de coagulação, eventos cardiovasculares, lesões de pele, queimaduras, entre outros ${ }^{10-12}$.

Assim, a utilização de recursos de proteção, por meio de colchão de ar micropulsante, coxins ou almofadas, durante a permanência do paciente na mesa cirúrgica, promove o alívio da pressão, ademais dos objetivos de evitar atritos e prevenir lesões de pele, compressões ou distensões neuromusculares, queimaduras, entre outros danos ${ }^{11,12}$.

A prescrição de enfermagem é um conjunto de ações ou intervenções determinadas pelo enfermeiro com o intuito de atingir resultados esperados no paciente, para prevenir, proteger, promover, recuperar e manter sua saúde ${ }^{1}$. Assim, fica evidente sua importância na assistência de enfermagem e no desenvolvimento da SAEP, independentemente do período perioperatório no qual o paciente se encontra.

Os registros de enfermagem são essenciais para o desenvolvimento da qualidade da assistência de enfermagem, validando o cuidado prestado pela equipe, tendo em vista a continuidade da assistência de forma individualizada e planejada, bem como a segurança do paciente e da equipe que lhe assiste. Estudos evidenciam que, embora os registros constituam a única forma de analisar o cuidado profissional, comprovar e validar a prática da equipe de enfermagem, há uma deficiência na atuação da enfermagem perante as anotações ou os registros, assim como na qualidade desses registros, observando-se anotações incompletas, dificultando a análise da auditoria de enfermagem ${ }^{7,13}$.

Verificou-se, no pós-operatório, a deficiência dos registros em relação à prescrição de enfermagem e também à avaliação da assistência prestada. Assim, ficou claro o maior enfoque dos profissionais no que tange ao registro dos cuidados ligados ao procedimento médico, em detrimento dos cuidados acerca do conforto do paciente, prejudicando a continuidade e a qualidade da assistência prestada e do PE. Da mesma maneira, observou-se que o registro sobre os equipamentos e materiais também foi priorizado. Segundo estudo realizado por meio de revisão narrativa, os registros de enfermagem estão sendo feitos com abreviaturas, de forma reduzida, incompleta e com conteúdo que privilegia o saber biomédico ${ }^{14}$. 
No CC, com base nas anotações de enfermagem e nos cuidados prescritos, é justificada a utilização de equipamentos, materiais e medicamentos. Os débitos devem ser assinalados na nota de gastos. Logo, os erros em cobranças estão relacionados à falha nos registros por parte das equipes tanto de enfermagem quanto médicas. Logo, as anotações de enfermagem nos prontuários são muito importantes para a instituição e utilizadas pelo processo de auditoria com o objetivo de apontar inadequações na assistência no tocante aos serviços prestados, realizar o faturamento das contas e rever as glosas ${ }^{13,15}$.

No pós-operatório, com base na prescrição de enfermagem, a equipe de enfermagem deve fornecer cuidados intensivos e semi-intensivos aos pacientes, direcionados à recuperação da consciência, à estabilização dos sinais vitais, à motilidade e à homeostase ${ }^{1}$. A visita pós-operatória de enfermagem corresponde à etapa que completa o PE no CC, momento no qual são avaliados as falhas e o sucesso dos cuidados prestados nos períodos pré e transoperatório, ou seja, a avaliação da assistência. Assim, a visita destaca-se como método de avaliação da assistência prestada, empenhando-se em seguir os requisitos de qualidade segundo o paciente ou os objetivos técnicos estabelecidos ${ }^{4}$.

Estudos revelam as dificuldades do enfermeiro quanto à implementação da $\mathrm{SAE}$, justificadas por sobrecarga de trabalho, desvio de função, falta de entendimento e conhecimento dos próprios profissionais ou motivos institucionais ${ }^{16,17}$. Conforme pesquisas ${ }^{2}$, a baixa adesão à SAEP pode estar relacionada à baixa procura de atualização acerca do tema pelos profissionais e à desmotivação e falta de estímulo por parte da instituição. Além disso, o mesmo estudo demonstrou que apenas uma etapa da SAEP havia sido realizada, sendo esta a visita pré-operatória.

Destaca-se a importância da conscientização do enfermeiro no tocante à necessidade dos registros de enfermagem e da implementação da SAE. O enfermeiro deve evitar as atividades que não são de sua competência, empenhando-se por melhores condições de trabalho, que permitam executar os registros de enfermagem, tornando a sistematização ativa ${ }^{16}$.

Nesse contexto, torna-se necessário que os profissionais se atualizem constantemente, tendo em vista a qualificação do raciocínio clínico e do crítico, essenciais à aplicação do $\mathrm{PE}$, garantindo um cuidado seguro e de qualidade. É importante que os profissionais de enfermagem atuantes no CC se proponham a implementar diária e adequadamente o $\mathrm{PE}$, visto que, em alguns casos, esse instrumento é aplicado de forma fragmentada e desconectada da realidade ${ }^{18}$.

Vale ressaltar que a implementação da SAE e da SAEP é uma exigência do Conselho Federal de Enfermagem e que "os registros respaldam o cuidado de enfermagem prestado ao paciente no CC” ${ }^{\prime 1}$. Conforme a Lei n ${ }^{\circ} 8.078^{19}$, compete ao profissional e/ou à instituição comprovar a realização de assistência adequada ao paciente, livre de riscos e danos.

\section{CONCLUSÃO}

Conforme as recomendações da SOBECC para atuação do enfermeiro no perioperatório, com base na análise dos registros de enfermagem, observou-se deficiência nos registros e na adesão à SAEP, considerando a alta tecnologia disponível no mercado atualmente e as exigências da legislação.

Ficou evidente que os cuidados relacionados aos procedimentos médicos foram os mais registrados, em detrimento do registro dos cuidados pertinentes ao conforto do paciente. Da mesma forma, constatou-se que os registros acerca dos equipamentos e materiais também foram priorizados.

Ressalta-se como limitação deste estudo o fato de estar baseado somente em registros, e não em observações diretas. Logo, não é possível concluir se faltou a realização do procedimento ou somente de seu registro.

Assim, sinaliza-se para a importância de se fazer os registros de enfermagem, bem como a necessidade da qualificação profissional, tendo em vista o respaldo legal do trabalho do enfermeiro, a satisfação e o reconhecimento profissional, contribuindo para melhor qualidade da assistência de enfermagem aos pacientes e à família.

Vale destacar a necessidade dos enfermeiros de priorizar o desenvolvimento dos cuidados aos pacientes e familiares, além das demais atribuições específicas da enfermagem, rejeitando as atividades que não são de sua competência, levando em conta a validação do PE e a implementação da SAE. 


\section{REFERÊNCIAS}

1. Associação Brasileira de Enfermeiros de Centro Cirúrgico, Recuperação Anestésica e Centro de Material e Esterilização (SOBECC). Diretrizes de práticas em enfermagem cirúrgica e processamento de produtos para a saúde. 7a ed. São Paulo: SOBECC; Barueri: Manole; 2017.

2. Ribeiro E, Ferraz KMC, Duran ECM. Atitudes dos enfermeiros de centro cirúrgico diante da sistematização da assistência de enfermagem perioperatória. Rev SOBECC. 2017;22(4):201-7. http://doi.org/10.5327/ Z1414-4425201700040005

3. Sena AC, Nascimento ERP, Maia ARCR, Santos JLG. Construção coletiva de um instrumento de cuidados de enfermagem a pacientes no pré-operatório imediato. Rev Baiana Enferm. 2017;31(1):e20506. http://doi.org/10.18471/rbe.v31i1.20506

4. Carvalho R, Bianchi ERF. Enfermagem em Centro Cirúrgico e Recuperação Anestésica. 2a ed. São Paulo: Manole; 2016.

5. Adamy EK, Tosatti M. Sistematização da Assistência de Enfermagem no período perioperatório: visão da equipe de enfermagem. Rev Enferm UFSM [Internet]. 2012 [acessado em 10 maio 2018];2(2):30010. Disponível em: https://periodicos.ufsm.br/reufsm/article/ view/5054/3754

6. Fonseca RMP, Peniche ACG. Enfermagem em centro cirúrgico: trinta anos após criação do Sistema de Assistência de Enfermagem Perioperatório. Acta Paul Enferm. 2009;22(4):428-33. http://dx.doi. org/10.1590/S0103-21002009000400013

7. Azevedo LMN, Oliveira AG, Malveira FAS, Valença CN, Costa EO, Germano RM. A visão da equipe de enfermagem sobre seus registros. Rev Rene. 2012;13(1):64-73.

8. Brasil. Ministério da Saúde. Conselho Nacional de Saúde. Resolução no 466, de 12 de dezembro de 2012. Diretrizes e normas regulamentadoras de pesquisas envolvendo seres humanos [Internet]. Brasil: Ministério da Saúde; 2013 [acessado em 10 maio 2018]. Disponível em: http://bvsms. saude.gov.br/bvs/saudelegis/cns/2013/res0466_12_12_2012.html

9. Freiberger MF, Mudrey ES. A importância da visita pré-operatória para sistematização da assistência de enfermagem perioperatória. Rev Ciên Fac Edu Mei Amb. 2011;2(2):1-26. https://doi.org/10.31072/ rcf.v2i2.96

10. Danczuk RFT, Nascimento ERP, Silveira NR, Hermida PMV, Rasía MA. Métodos de aquecimento na prevenção da hipotermia no intraoperatório de cirurgia abdominal eletiva. Esc Anna Nery Rev Enferm. 2015;19(4):578-84. http://doi.org/10.5935/1414-8145.20150077

11. Miranda AB, Fogaça AR, Rizzetto M, Lopes LCC. Posicionamento cirúrgico: cuidados de enfermagem no transoperatório. Rev SOBECC. 2016;21(1):52-8. http://doi.org/10.5327/Z1414-4425201600010008

12. Bisinotto FMB, Dezena RA, Martins LB, Galvão MC, Martins Sobrinho J, Calçado MS. Queimaduras relacionadas à eletrocirurgia: relato de dois casos. Rev Bras Anestesiol. 2017;67(5):527-34. http://doi. org/10.1016/j.bjan.2016.03.003

13. Oliveira DR, Jacinto SM, Siqueira CL. Auditoria de enfermagem em centro cirúrgico. RAS [Internet]. 2013 [acessado em 10 maio 2018];15(61):151-8. Disponível em: http://www.cqh.org.br/portal/ pag/anexos/baixar.php?p_ndoc $=1021 \&$ p_nanexo=506

14. Araujo MM, Diniz SOS, Silva PS. Registros de enfermagem: reflexões sobre o cotidiano do cuidar. ABCS Health Sci. 2017;42(3):161-5. http:// doi.org/10.7322/abcshs.v42i3.920

15. Setz VG, D'Innocenzo M. Avaliação da qualidade dos registros de enfermagem no prontuário por meio da auditoria. Acta Paul Enferm. 2009;22(3):313-7. http://dx.doi.org/10.1590/ S0103-21002009000300012

16. Melo DFF, Nunes TAS, Viana MRP. Percepção do enfermeiro sobre a implantação da sistematização da assistência de enfermagem no centro cirúrgico. Rev Interd [Internet]. 2014 [acessado em 6 jun. 2018];7(2):36-44. Disponível em: https://revistainterdisciplinar. uninovafapi.edu.br/index.php/revinter/article/view/425/pdf_126

17. Botelho J, Veloso GBL, Favero L. Sistematização da assistência de enfermagem: o conhecimento da equipe de enfermagem de um centro cirúrgico. Enferm Foco. 2013;4(3-4):198-201. http://doi. org/10.21675/2357-707X.2013.v4.n3/4.552

18. Riegel F, Oliveira Jr. NJ. Processo de enfermagem: implicações para a segurança do paciente em centro cirúrgico. Cogitare Enferm. 2017;22(1):1-5. http://doi.org/10.5380/ce.v22i1.45577

19. Brasil. Lei $n^{\circ} 8.078$, de 11 de setembro de 1990. Dispõe sobre a proteção do consumidor e dá outras providências. Diário Oficial da União [Internet]. 2017 [acessado em 7 jun. 2018];Seção 1 (Supl.). Disponível em: http://www2.camara.leg.br/legin/fed/lei/1990/lei8078-11-setembro-1990-365086-publicacaooriginal-1-pl.html 